\title{
Nachtrag während der Korrektur
}

\section{zur Arbeit Mestitz: „Zur Frage der Beziehungen zwischen Keimdrüsen und Geschlechtsmerkmalen" (d. Heft, S. 662).}

Nach Fertigstellung der Arbeit sind noch verschiedene Beiträge erschienen, die auf unser Thema Bezug haben und die wir kurz erwähnen möchten:

Orsós (Dtsch. Z. Chir. 230 (1931)] beschreibt einen Fall von Pseudohermaphroditismus mit eindeutig weiblichen sekundären Geschlechtsmerkmalen trotz Gegenwart von Hoden mit reichlich interstitiellem Gewebe. Auf Grund dieses Befundes tritt er für die Auffassung Halbans und gegen die Anschauung Steinachs ein.

Krediet [Z. Anat. 94 (1931)] teilt 2 weitere Fälle von lateralem Hermaphroditismus beim Schwein mit. Auch nach diesem Autor sind die Halbseitenzwitter keineswegs Hormonintersexe. Die Entscheidung, ob es sich um Raumintersexe (Gynandromorphe) oder Zeitintersexe handelt, ist allerdings schwierig; wahrscheinlicher soll die letztere Annahme sein, doch stößt auch sie auf Schwierigkeiten, da nicht zu erklären ist, warum wohl die eine Gonade ihr Geschlecht verändert hat, die andere aber nicht.

Ähnlich sprechen sich Moszkowicz und Kolmer [Arch. path. Anat. u. Physiol. 279 (1931)] gelegentlich der Schilderung eines Huhnes mit Halbseitenhermaphroditismus aus. Das Vorkommen dieser Anomalie ,,ist ein klarer Beweis dafür, daß der geschlechtliche Dimorphismus der Tiere nicht allein durch die Hormonwirkung der Keimdrüse bedingt sein kann. Denn hier haben wir beide Keimdrüsen in einem Organismus nebeneinander und dennoch zeigen beide Körperhälften genau bis zur Mittellinie verschiedene Geschlechtsmerkmale. Das kann nur so erklärt werden, daß die einzelnen Zellen der beiden Körperhälften einen geschlechtlichen Unterschied aufweisen".

In ausgedehnten Untersuchungen gelangte H. O. Neumann [Z. Geburtsh. 99 (1930), Sitzgsber. Ges. Naturwiss. Marburg 1930] zu einer Bestätigung der von Halban festgestellten Schwangerschaftsreaktion fetaler Organe und konnte diese Befunde dahin erweitern, daß eine solche Reaktion auch an der Vagina des neugeborenen Mädchens nachzuweisen ist. Die Befunde an den Hoden sind nicht einheitlich, "teilweise sieht man Rückbildungsvorgänge, teilweise aber auch Zeichen einer fortschreitenden Entwicklung“. 
Zur Frage der antagonistischen Wirkung zwischen männlicher und weiblicher Keimdrüse: Migliavacca [Ber. Gynäk. 19 (1931)] fand, daß Hodenextrakte in geringen Dosen sogar stimulierend und erst in höheren lähmend auf das Ovarium wirken. - Hingegen soll es Reiprich [Münch. med. Wschr: 9 (1931)] gelungen sein, männliche Versuchstiere durch Implantation von Ovarium ,hormonal“" zu sterilisieren.

Probstner [Endokrinol. 8 (1931)] veröffentlicht eine den Fällen Waldsteins, Amatis und Szarkas analoge Beobachtung von Kastration einer Schwangeren am 53. Tage nach Beginn der letzten Menstruation. Der Schwangerschaftsverlauf war ungestört bei normaler Hormonbilanz; der Autor erblickt darin eine neuerliche Bestätigung der Annahme Halbans von der innersekretorischen Tätigkeit der Placenta.

Zum Schluß noch einige Worte zum Kapitel „Geschlechtsbeeinflussung durch Tumoren": Der Freundlichkeit des Herrn Prim. Dr. Jung in St. Gallen, auf dessen Abteilung der von uns zitierte Fall Wetterwalds von postklimakterischer Blutung beobachtet wurde, verdanken wir die ausführliche Mitteilung der Krankengeschichte, aus der hervorgeht, daß keinerlei Anzeichen einer Vermännlichung bestanden. Herr Prof. Helly hat uns in liebenswürdigster Weise einen Schnitt des Tumors zur Verfügung gestellt, den wir mit seinem Einverständnis Herrn Prof. $R$. Meyer zur Begutachtung vorgelegt haben. Prof. Meyer äußert sich dahin, daß, sofern aus dem nicht ausreichenden Material eine Diagnose möglich ist, wohl nicht ein tubuläres Adenom, sondern eher ein Granulosazelltumor vorliegen dürfte, an dem allerdings der große Reichtum an Strängen und Schläuchen außergewöhnlich ist. - Es beweist ${ }^{1}$ dies wieder nur,

1 Anmerkung von Robert Meyer: Die Worte von Mestitz „es beweist hier wieder nur, wie wenig geklärt der großeFragenkomplex noch ist" Der Fragenkomplex ist zwar selbstverständlich nicht nach allen Richtungen geklärt, aber der Fall Helly ist nicht von Belang für ihn, vielmehr ist dieser Fall von Granulosatumor Hellys nur insofern interessant, als er in dem einzigen kleinen mikroskopischen Schnitte, der mir vorgelegen hat, sehr viele Schläuche enthält, aber nur der Menge nach mehr, als man sonst sieht. Nur am Rande des Schnittes und an wenigen kleinen Stellen konnte ich erkennen, daß es sich um einen Granulosazelltumor handelte. Namentlich fiel eine basale Zellschicht an einem offenbar größeren aber nur eben angeschnittenen Granulosazellhaufen auf und an einer Stelle die typische Verwicklung der Zellstränge. Ich habe nur gewünscht, daß mehr Material zur Untersuchung gestellt würde, aber solches war leider nicht mehr vorhanden. Herr Kollege Helly hat mir jedoch zugesagt, daß er mir den Tumor senden würde, falls er sich noch findet. Trotz dieses Mangels ist für mich kein Zweifel, daß der Tumor dem Granulosaepithel angehört. Besonders hervorzuheben ist, daß diese Deutung nicht vorbeeinflußt war, da ich weder die Vorgeschichte noch überhaupt die Herkunft (Helly) kannte. - Die ausgiebigere Untersuchung solcher Fälle würde jedoch sehr erwünscht sein, nicht nur weil es die Kenntnis der wechselvollen Strukturen der Granulosazelltumoren bereichern würde, sondern weil die von mir aufgeworfene Frage noch offen steht, ob möglicherweise 2 Arten von Tumoren miteinander oder durcheinander wachsen können. Dieses bezieht 
wie wenig geklärt der ganze Fragenkomplex noch ist, was auch noch aus folgender Mitteilung hervorgeht:

Tietze [Zbl. Gynäk. 9 (1931)] berichtet über 3 Fälle atypischer, an die Andreioblastome Meyers erinnernden Tumoren, die wohl eine hyperplasierende Wirkung auf den übrigen Genitalapparat, also einen fördernden Einfluß auf die isosexuellen Merkmale hatten, aber von keinerlei Symptomen einer Entweiblichung oder gar Vermännlichung begleitet waren.

Die in allerletzter Zeit in Pflügers Arch. 22\% (1931) veröffentlichten Versuche von Steinach und Kun - Vermännlichung durch Corpus luteum! - bilden schließlich eine weitere, wertvolle Bestätigung dessen, daß wir nicht berechtigt sind, eine geschlechtsspezifische Wirkung der Keimdrüsenhormone anzunehmen.

sich zwar hauptsächlich auf Mischungen mit den Digerminomen, denn eine Mischung von Tumoren aus indifferentem Zellmaterial und aus sexuellem Zellmaterial würde ich für viel eher möglich halten als Gynandroblastome, wegen der von mir angenommenen sexuellen Gegensätzlichkeit. - Als ebenso wichtig - auch das habe ich schon betont - würde ich es begrüßen, wenn man den Arrhenoblastomen morphologisch ähnliche oder gleichartige Tumoren auffinden würde, die keine Erscheinungen von Vermännlichung hervorrufen, weil solche Fälle zeigen würden, daß nicht jede Person auf die Tumoren gleichartig reagiert. Diese Fälle lassen sich — glaube ich - möglicherweise finden, weil die Reaktion der Vermännlichung an den bekannten Fällen individuell verschiedene Grade zeigt und weil sie beim Adenoma tubulare testiculare scheinbar des öfteren fehlt. Es soll sich dann erweisen, ob der Unterschied der Reaktion in Verschiedenheiten der Tumoren oder der Trägerinnen beruht. - Der Fall Helly ist nach meiner Deutung ein Granulosazelltumor und die mir nachträglich bekanntgewordene Anamnese spricht auch dafür. Ich kann mir zwar denken, daß „Arrhenoblastome“ zur Vermännlichung nicht in jedem Falle führen müssen, aber es würde einen unerklärlichen Widerspruch bedeuten, wenn ihr sonst stets zur Entweiblichung, mindestens zur funktionellen Atrophie des Ovarium führender hormonaler Einfluß eine Umkehr dahin erfahren sollte, daß eine Hypertrophie des Endometrium oder des Uterus entstünde wie bei den Granulosazelltumoren. - Im übrigen verzichten die an diesem Gebiete besonders beteiligten Herausgeber $G$. A. Wagner und $R$. Meyer auf eine Kritik der Einzelheiten in dem vorstehenden Aufsatze von Mestitz. 